Arch. Tierz., Dummerstorf 50 (2007) 6, 619-627

Aus dem Institut für Agrar- und Ernährungswissenschaften der Martin-Luther-Universität Halle-Wittenberg

NORBERT MIELENZ und LUTZ SCHÜLER

\title{
Konstruktion von Indizes mit Restriktionen in Random Regression Modellen zur Veränderung der Wachstumskurve
}

\begin{abstract}
Title of the paper: Index construction with restrictions in random regression models to change the pattern of the growth curve

Random regression models provide estimated breeding values (EBV) for the complete growth curve for any target age. The animal-specific curves can be described as the weighted sum of continuous covariates with random regression coefficients. By using the covariance matrix $\mathrm{K}$ of the additive genetic regression coefficients the response to index selection can be calculated for any age or time of the test period. In this study selection indexes with equality restrictions based on the eigenvectors of matrix K were used to modify the growth curve of the population. In order to demonstrate the index construction a matrix $\mathrm{K}$ was used, estimated from repeated measurements for body weight of bulls by using Legendre polynomials as covariates. Indexes for high and low growth rate until age at the reflection point were derived subject to the restriction of zero gain for initial and final body weight. Selection strategies for improving body weight at the end of the test period while holding the daily gain in a certain time interval on a desired level were compared. By using so-called "restrictive economic values”, an aggregate breeding value for body weight was derived from EBV for individual growth curve.
\end{abstract}

Key Words: growth curve, eigenvector index, restrictions

\section{Zusammenfassung}

Random Regression Modelle liefern geschätzte Zuchtwerte für die vollständige Wachstumskurve zu jedem Alter innerhalb der Prüfperiode. Die tierspezifischen Kurven können als die mit zufälligen Regressionskoeffizienten gewichtete Summe von stetigen Kovariablen dargestellt werden. Unter Verwendung der Kovarianzmatrix K der additiv-genetischen Regressionskoeffizienten kann die genetische Veränderung bei Indexselektion für beliebiges Alter oder zu beliebigen Zeitpunkten der Prüfperiode berechnet werden. In dieser Studie werden Selektionsindizes mit Gleichungsrestriktionen basierend auf den Eigenvektoren der Matrix K zur Modifikation der Wachstumskurve in der Population abgeleitet. Zur Demonstration der Indexkonstruktion wird eine Matrix K genutzt, welche an wiederholt erfassten Körpergewichten von Bullen mit Hilfe von Legendre Polynomen als Kovariablen geschätzt wurde. Selektionsindizes zur Erhöhung bzw. zur Verringerung der Wachstumsgeschwindigkeit bis zum Wendepunkt bei unverändertem Anfangs- und Endgewicht werden angegeben. Das Konzept der restriktiven Indizes wird genutzt, um Zuchtwerte für hohes Endgewicht bei vorgegebener suboptimaler Erhöhung der täglichen Zunahme in bestimmten Zeitintervallen aufzustellen. Durch die Bestimmung von „restriktiven ökonomischen Gewichten“ zu ausgewählten Zeitpunkten lässt sich aus den geschätzten Zuchtwertkurven des Wachstums ein Gesamtzuchtwert für das Körpergewicht ableiten.

Schlüsselwörter: Wachstumskurve, Eigenvektorindex, Restriktionen

1. Einleitung

Bei Vorliegen von wiederholten Beobachtungen pro Tier für dasselbe Merkmal erfolgt die Zuchtwertschätzung (ZWS) zunehmend mit Random Regression Modellen (RRM), welche von SCHAEFFER und DEKKERS (1994) sowie von JAMROZIK und SCHAEFFER (1997) in die Tierzucht eingeführt wurden. Im Ergebnis der ZWS werden pro Tier mehrere zufällige additiv-genetische Regressionskoeffizienten verknüpft mit zeit- bzw. altersabhängigen Kovariablen geschätzt (KREJCOVA et al., 2007b). 
Folglich lassen sich pro Tier nicht nur Zuchtwerte zu bestimmten Zeitpunkten sondern über den Beobachtungszeitraum hinweg Zuchtwertkurven ausweisen. Die Variabilität dieser Kurven in der Population kann auf die Variabilität der zufälligen Regressionskoeffizienten zurückgeführt werden, wobei deren Variabilität wiederum durch die Varianz-Kovarianz Matrix (K) der additiv-genetischen Regressionskoeffizienten beschrieben werden kann. Schätzungen für die Matrix K und für die daraus abgeleiteten Kovarianzfunktionen auf der Grundlage von wiederholt erfassten Körpergewichten mit Hilfe von Polynomansätzen findet man beispielsweise bei MEYER (1999, 2001); bei ALBUQUERQUE und MEYER (2001) und bei ARANGO et al. (2002). Durch die Bestimmung der Eigenwerte und -vektoren der Matrix K lassen sich Aussagen über mögliche genetische Veränderungen eines Merkmals über den gesamten Beobachtungszeitraum hinweg gewinnen (KIRKPATRICK et al., 1990). Der Züchter steht folglich vor der Aufgabe anhand des Verlaufs der tierspezifischen Zuchtwertkurven über bestimmte Zeitabschnitte hinweg oder anhand der Zuchtwerte berechnet aus den Kurven zu exponierten Zeitpunkten die Selektion der Tiere durchzuführen. In der Milchproduktion ist beispielsweise neben der Laktationsleistung die Persistenz als ökonomisch wichtiges Merkmal im Zuchtziel zu berücksichtigen (SWALVE and GUO, 1999; TOGASHI and LIN, 2006). Bei der Selektion von Fleischrindern ist neben hohem Endgewicht zusätzlich die tägliche Zunahme in einem bestimmten Zeitabschnitt vorrangig zu verbessern. Zur Erreichung der oben formulierten Zuchtziele kann es notwendig sein die Gestalt der Laktations- bzw. der Wachstumskurve in der Population genetisch zu verändern. Dazu kann die Indextheorie von HAZEL (1943) dienen, indem die Eigenvektoren, deren Dimension der Anzahl der Regressionskoeffizienten entspricht, in einem Selektionsindex linear kombiniert werden. Bestehen nur unklare Vorstellungen über die ökonomische Bewertung von Merkmalsausprägungen zu verschiedenen Zeitpunkten und Zeitabschnitten, so kann das Konzept der restriktiven Indizes zur Überprüfung und zur Ableitung von Zuchtzielen verwendet werden (KEMPTHORNE and NORDSKOG, 1959; HARVILLE, 1974; LIN and TOGASHI, 2005). Ziele dieser Arbeit sind: a) Darstellung von Möglichkeiten zur genetischen Veränderung der populationsspezifischen Wachstumskurve b) exemplarische Ableitung von Zuchtwerten für das Körpergewicht unter Einbeziehung der täglichen $\mathrm{Zu}$ nahme aus bestimmten Zeitabschnitten.

2. Material und Methoden

2.1. Berechnung der Selektionserfolge

Sei $\alpha=\left(\alpha_{0}, \cdots, \alpha_{k-1}\right)^{\prime}$ ein Vektor von additiv-genetischen Regressionskoeffizienten zugehörig zu Kovariablen, die durch Legendre Polynome vom Grad 0 bis (k-1) gegeben sind. Weiter sei $K$ die Varianz-Kovarianz Matrix (VC-Matrix) von Vektor $\alpha$, dann besitzt $K$ die Zerlegung:

$$
\begin{aligned}
& E^{\prime} K E=\Lambda \text { und } E^{\prime} E=I \\
& \operatorname{mit} \Lambda=\operatorname{Diag}\left(\lambda_{i}\right)
\end{aligned}
$$

Hierbei sind $\lambda_{\mathrm{i}}(i=1, \ldots, k)$ die Eigenwerte von $K$ und $E$ eine $(k \times k)$-Matrix, deren Spalten aus den orthogonalen Eigenvektoren $e_{\mathrm{i}}$ zugehörig zu den Eigenwerten $\lambda_{\mathrm{i}}$ bestehen. Bezeichne $\phi_{m}\left(t_{s}\right)$ das Legendre Polynom vom Grad $m$ standardisiert auf das Intervall [-1,1] zum Zeitpunkt $t_{\mathrm{s}}$. Dann besitzt der Zuchtwert eines Tieres zum Zeitpunkt $t$ die Darstellung: 


$$
g(t)=\sum_{m=0}^{(k-1)} \phi_{m}\left(t_{s}\right) \cdot \alpha_{m}=\left(\phi_{0}, \cdots, \phi_{k-1}\right)^{\prime} \alpha=\phi^{\prime} \alpha
$$

Seien $g=\left(g\left(t_{1}\right), \cdots, g\left(t_{N}\right)\right)^{\prime}$ der Vektor von genetischen Effekten eines Tieres und $\Phi$ die zugehörige $(N \times k)$-Matrix von Kovariablen gegeben durch Legendre Polynome jeweils berechnet zu den Zeitpunkten $t_{1}$ bis $t_{\mathrm{N}}$. Dann gilt:

$$
\begin{aligned}
& g=\Phi \alpha \text { und } \operatorname{Var}(g)=G=\Phi K \Phi^{\prime} \\
& \text { mit } \operatorname{Rg}(G)=\operatorname{Rg}(K)=k
\end{aligned}
$$

Da die Matrix $G$ nicht vollen Rang besitzt, muss die Indexkonstruktion auf den Eigenvektoren der Matrix K basieren. Gemäß der Indextheorie von HAZEL (1943) werden für den Selektionsindex und das Zuchtziel die folgenden Ansätze gewählt (s. TOGASHI and LIN, 2006).

$$
I=\sum_{i=1}^{k} b_{i} \cdot\left(\alpha^{\prime} e_{i}\right)=b^{\prime}\left(E^{\prime} \alpha\right) \quad \text { und } \quad H=\sum_{i=1}^{N} a_{i} \cdot g_{i}=a^{\prime} \Phi \alpha
$$

Unter der Annahme, dass die genetischen Effekte in Darstellung (3) bekannt sind, ergibt sich eine Korrelation zwischen I und $\mathrm{H}$ von Eins, falls gilt:

(5)

$$
b=E^{\prime} \Phi^{\prime} a \quad \text { bei } \quad \Phi^{\prime} a \neq 0
$$

Setzt man für den Selektionsindex und die Zuchtwerte jeweils zweidimensionale Normalverteilung voraus und bezeichnet man mit $d_{\mathrm{s}}$ die standardisierte Selektionsdifferenz, dann besitzen die partiellen Selektionserfolge (SE) die Gestalt:

$$
\Delta g=\frac{\operatorname{cov}(I, g)}{\sigma_{I}^{2}} \cdot \Delta I=\frac{d_{s}}{\sigma_{I}} \cdot \Phi K E b \quad \text { mit } \quad \sigma_{I}^{2}=b^{\prime}\left(E^{\prime} K E\right) b=b^{\prime} \Lambda b
$$

\section{2 .}

Indexkonstruktion mit Restriktionen

Die Vorgabe von ökonomischen Gewichten im Zuchtziel $H$ kann sich als schwierig erweisen oder unerwünschte genetische Veränderungen in den partiellen Selektionserfolgen hervorrufen. Deshalb stellt die Maximierung des Gesamtselektionserfolges $\Delta H=a^{\prime} \Delta g$ unter Restriktionen auf die partiellen Selektionserfolge eine mögliche Alternative dar. Zur Formulierung von Gleichungsrestriktionen wird gesetzt:

$$
\begin{aligned}
& m_{i}^{\prime} \Delta g=d_{i}^{*} ; \quad i=1, \cdots, n \\
& \text { bzw. } M^{\prime} \Delta g=d^{*} \quad \text { mit } \quad n=R g(M) \leq k
\end{aligned}
$$

Hierbei ist $\mathrm{M}$ eine $(\mathrm{N} \times \mathrm{n})$-Matrix, deren Spalten die Vektoren $m_{\mathrm{i}}$ sind. Enthält $m_{\mathrm{i}}$ neben Nullen genau eine Eins, so ist ein partieller SE fest vorgegeben. Die Vektoren $m_{\mathrm{i}}$ können aber auch mehrere von Null verschiedene Elemente besitzen. In diesem Fall wird eine Linearkombination von SE fest vorgegeben. Folglich ist $d^{*}=\left(d_{1}^{*}, \cdots, d_{n}^{*}\right)^{\prime}$ ein Vektor von Vorgabewerten für partielle SE oder deren Linearkombinationen. Aufgrund der Darstellungen (2) und (6) lassen sich die partiellen SE durch Polynome vom Grad (k-1) beschreiben. Folglich ist nur die Vorgabe von maximal (k-1) unabhängigen Restriktionen möglich.

Die optimalen restriktiven Indexgewichte ergeben sich durch Lösung des folgenden Optimierungsproblems.

$$
\begin{array}{ll}
c_{0}^{\prime} b \rightarrow \underset{b}{M a x !} \text { bei } \quad C^{\prime} b=d \quad \text { und } \quad b^{\prime} \Lambda b=1 \\
\text { mit } \quad d=\left(1 / d_{s}\right) \cdot d^{\bullet} ; \quad c_{0}^{\prime}=a^{\prime} \Phi K E ; \quad C^{\prime}=M^{\prime} \Phi K E
\end{array}
$$

Eine Lösung von (8) existiert unter folgenden Bedingungen:

(a) Die Matrix $\left(M^{\prime} G M\right)^{-1}$ existiert, es gilt also $n=R g(M) \leq k$ 
(b) $\quad d^{\prime}\left(M^{\prime} G M\right)^{-1} d \leq 1 \quad$ und $\quad R g(M, a)=R g(M)+1$

Unter Verwendung der Lagrange-Multiplikatoren Methode ergibt sich für Optimierungsproblem (8) die folgende Lösung (vgl. HARVILLE, 1974):

$$
\begin{aligned}
& b=E^{\prime} \Phi^{\prime} M\left(M^{\prime} G M\right)^{-1} d+\beta_{0} \cdot P c_{0} \\
& \text { mit } P c_{0}=E^{\prime} \Phi^{\prime}\left[I-M\left(M^{\prime} G M\right)^{-1} M^{\prime} G\right] a \\
& \beta_{0}=\frac{\left[1-d^{\prime}\left(M^{\prime} G M\right)^{-1} d\right]^{\frac{1}{2}}}{\left(c_{0}^{\prime} P c_{0}\right)^{\frac{1}{2}}}
\end{aligned}
$$

Bemerkung 1: Führt man in Darstellung (9) den Lagrange'schen Multiplikator $\beta$ ein, so besitzt der optimale Lösungsvektor $b$ die Darstellung (CUNNINGHAM et al., 1970; NIEBEL and VAN FLECK, 1983).

$$
\begin{aligned}
& \beta=\left(M^{\prime} G M\right)^{-1}\left(M^{\prime} G a-\beta_{0}^{*} \cdot d\right) \text { mit } \beta_{0}^{*}=\left(1 / \beta_{0}\right) \\
& b=\beta_{0} \cdot E^{\prime} \Phi^{\prime}(a-M \beta)
\end{aligned}
$$

Vergleicht man die Darstellung in (10) mit der Formel (5), so besitzen die „restriktiven ökonomischen Gewichte“ die Gestalt:

$$
a^{*}=(a-M \beta)
$$

Die Indexgewichte $b^{*}=E^{\prime} \Phi^{\prime} a^{*}$ mit $a^{*}=\left(a_{1}^{*}, \cdots, a_{N}^{*}\right)^{\prime}$ liefern folglich dieselben Selektionserfolge wie der Index mit Restriktionen.

Bemerkung 2: Bezeichne $I_{e(i)}=e_{i}^{\prime} \alpha$ einen Index, der eine Linearkombination der Regressionskoeffizienten gewichtet mit den Elementen des $i$-ten Eigenvektors von Matrix K darstellt. Die Indizes $I_{e(1)}$ bis $I_{e(k)}$ repräsentieren unabhängige Merkmale, deren Varianzen den Eigenwerten der Matrix K entsprechen. Folglich gilt:

$$
\frac{\operatorname{Var}\left(I_{e(i)}\right)}{\operatorname{Var}\left(I_{e(1)}+\cdots+I_{e(k)}\right)}=\frac{\lambda_{i}}{\sum_{i} \lambda_{i}}
$$

Mit Hilfe der Eigenwerte kann somit der Anteil der einzelnen Eigenvektoren an der genetischen Gesamtvariabilität veranschaulicht werden.

Bemerkung 3: Sei $b^{i}$ ein $(\mathrm{k} \times 1)$-Vektor, der an Position $i$ eine Eins und ansonsten nur Nullen als Elemente enthält. Dann besitzen die k separaten Indizes aus Eigenvektoren die Gestalt:

$$
I_{e(i)}=e_{i}^{\prime} \alpha=\left(b^{i}\right)^{\prime} E^{\prime} \alpha
$$

Der Index (13) maximiert das Zuchtziel falls $E^{\prime} \Phi^{\prime} a=b^{i}$ gilt. Dieses Gleichungssystem ist nicht eindeutig lösbar. Zur Bestimmung der Nicht-Nullelemente von Vektor a müssen $\mathrm{k}$ Zeitpunkte $t_{1}$ bis $t_{\mathrm{k}}$ vorgegeben werden. Sei $\Phi_{r}$ eine $(\mathrm{k} \times \mathrm{k})$-Matrix, die aus $\Phi$ durch Streichen der Zeilen entsteht, die nicht zu $t_{1}$ bis $t_{\mathrm{k}}$ gehören. Dann gilt:

$$
a_{r}=\left(E^{\prime} \Phi_{r}\right)^{-1} b^{i}
$$

Wegen Darstellung (14) lässt sich zu jedem Index $I=b^{\prime} E^{\prime} \alpha$ ein Vektor von relativen ökonomischen Gewichten bestimmen, der nur zu k-Zeitpunkten von Null verschiedene Elemente besitzt.

\subsection{Numerisches Beispiel}

Die Indexkonstruktion mit Restriktionen als Hilfsmittel zur Veränderung der Wachstumskurve wird an genetischen Parametern von Fleischrindern demonstriert 
(MATTHES et al., 1996; KREJCOVA, et al., 2007a). Zur Zuchtwertschätzung mit einem Random Regression Modell standen im Zeitraum von 10 bis 420 Tagen pro Bulle durchschnittlich 8 Beobachtungen für das Körpergewicht zur Verfügung. Die additivgenetische VC-Matrix (K) der zufälligen Regressionskoeffizienten, geschätzt unter Verwendung von Legendre Polynomen 4-ten Grades, besitzt die folgende Gestalt:

$$
K=\left[\begin{array}{rrrrc}
400.914 & 157.906 & 1.167 & -10.916 & -2.735 \\
157.906 & 97.536 & 6.633 & -13.320 & -0.529 \\
1.167 & 6.633 & 7.086 & -2.399 & -0.932 \\
-10.916 & -13.320 & -2.399 & 7.479 & -1.593 \\
-2.735 & -0.529 & -0.932 & -1.593 & 2.78
\end{array}\right]
$$

Die Berechnung der Eigenwerte und -vektoren erfolgte mit dem Statistikprogramm SAS unter Verwendung der Matrixprogrammiersprache IML durch Aufruf der Funktionen eigval und eigvec. An der Summe aller Eigenwerte von Matrix K besitzen die Eigenwerte zugehörig zu den 5 orthogonalen Eigenwerten einen Anteil von 90.88, 6.61, 1.18, 1.03 und 0.31 Prozent.

Die Matrix $\Phi$ ist eine $(411 \times 5)$-Matrix, deren Zeilen durch die Werte der Legendre Polynome vom Grad 0 bis 4 an der Stelle $t_{\mathrm{s}}=f(t)$ mit $t=10,11, \ldots, 420$ gegeben sind. Im Beispiel gilt:

$$
t_{s}=f(t)=\frac{2 \cdot\left(t-t_{\min }\right)}{\left(t_{\max }-t_{\min }\right)}-1 \quad \text { mit } \quad t_{\min }=10 ; \quad t_{\max }=420
$$

Setzt man in (16) $t=10$ und t=420, so besitzen die Zeilen 1 und 411 in $\Phi$ die Gestalt:

$$
\begin{aligned}
& \Phi_{1}=\left[\begin{array}{lrrrr}
0.707 & -1.225 & 1.581 & -1.871 & 2.121
\end{array}\right] \\
& \Phi_{411}=\left[\begin{array}{lrrrr}
0.707 & 1.225 & 1.581 & 1.871 & 2.121
\end{array}\right]
\end{aligned}
$$

Die optimalen Indexgewichte beispielsweise für die Lösung des Problems

$$
\Delta g_{240} \rightarrow \text { Max! bei } \Delta \mathrm{g}_{10}=\Delta g_{420}=0
$$

können aus der Darstellung (9) abgeleitet werden. Durch entsprechende Wahl von M, d und a ergibt sich für den Vektor der Indexkoeffizienten zugehörig zu den 5 Eigenvektoren die folgende Darstellung:

(19) $\quad b=(0.010,-0.084,-0.292,-0.021,-0.350)^{\prime}$

Für den Vektor der von Null verschiedenen „restriktiven ökonomischen Gewichte“ gilt $a=\left(a_{10}, a_{240}, a_{420}\right)^{\prime}=(-0.216 ; 0.852 ;-0.477)^{\prime}$ falls $\|a\|^{2}=a^{\prime} a=1$ gefordert wird

3.

Ergebnisse

3.1. Modifikation der Wachstumskurve

Die Wachstumskurve in der Population bleibt unverändert durch den Einfluss von Selektion, falls die partiellen Selektionserfolge zu jeden Tag gleiche Veränderungen aufweisen. Da die SE sich als Funktion des Alters durch Polynome 4-ten Grades beschreiben lassen, müssen lediglich 4 Restriktionen an beliebigen voneinander verschiedenen Zeitpunkten vorgegeben werden. Beispielsweise kann man fordern:

$$
\begin{aligned}
& \Delta g_{420} \rightarrow \text { Max! } \\
& \text { bei } \quad \Delta \mathrm{g}_{80}=\Delta g_{160}=\Delta g_{240}=\Delta g_{320}=\Delta g_{400}
\end{aligned}
$$


Besteht dagegen der Wunsch zwei Teilpopulationen zu erzeugen, die sich in der Wachstumsgeschwindigkeit bis zum Wendepunkt $\left(t_{\mathrm{w}}\right)$ aber nicht im Anfangs- und Endgewicht unterscheiden, so kann man fordern:

$$
\begin{aligned}
& \Delta g\left(t_{W}\right) \rightarrow \text { Max! (bzw.Min!) } \\
& \text { bei } \quad \Delta g\left(t_{1}\right)=\Delta g\left(t_{N}\right)=0
\end{aligned}
$$

Die Ergebnisse der Indexkonstruktion mit Restriktionen gemäß (20) und (21), wobei $t_{1}=10, t_{\mathrm{w}}=240$ und $t_{\mathrm{N}}=420$ gesetzt wurde, enthält Tabelle 1 .

Tabelle 1

Partielle Selektionserfolge (in kg) in Abhängigkeit vom Zuchtziel H und von den Restriktionen (Genetic gains

\begin{tabular}{|c|c|c|c|c|c|c|c|}
\hline \multirow[b]{2}{*}{ Index } & \multirow[b]{2}{*}{$\mathbf{H}$} & \multirow[b]{2}{*}{ Restriktion } & \multicolumn{5}{|c|}{ partielle SE $\left(\right.$ mit $\left.d_{s}=1\right)$} \\
\hline & & & $\Delta \mathbf{g}_{10}$ & $\Delta g_{240}$ & $\Delta g_{420}$ & $\Delta \mathbf{g}_{\mathrm{TZ} 1}$ & $\Delta \mathbf{g}_{\mathrm{TZ} 2}$ \\
\hline $\mathrm{I}_{1 \mathrm{R}}$ & $\overline{g_{420}}$ & $\Delta \mathrm{g}(\mathrm{t})=\mathrm{const}$ & 7.68 & 7.68 & 7.68 & 0.0 & 0.0 \\
\hline $\mathrm{I}_{2 \mathrm{R}}$ & $\mathrm{g}_{240}$ & $\Delta \mathrm{g}_{10}=\Delta \mathrm{g}_{420}=0$ & 0.0 & 5.34 & 0.0 & 0.0232 & -0.0297 \\
\hline$I_{3 R}$ & $(-1) g_{240}$ & $\Delta \mathrm{g}_{10}=\Delta \mathrm{g}_{420}=0$ & 0.0 & -5.34 & 0.0 & -0.0232 & 0.0297 \\
\hline
\end{tabular}
depending on different breeding goals and restrictions)

Durch den Index $\mathrm{I}_{1 \mathrm{R}}$ wird die Wachstumskurve der Population zu jedem Zeitpunkt des Intervalls $[10,420]$ um einen konstanten Anteil nach oben verschoben. Die Indizes $I_{2 R}$ bzw. $I_{3 R}$ bewirken eine Erhöhung bzw. Verringerung der Wachstumsgeschwindigkeit bis zum 240. Tag, wobei die Körpergewichte in der Population zum 10. und zum 420. Tag unverändert bleiben.

\subsection{Körpergewichte und tägliche Zunahmen}

Die Forderung nach maximaler täglicher Zunahme in einem bestimmten zeitlichen Intervall muss nicht zwangsläufig auch das höchste Körpergewicht zum Ende der Prüfperiode hervorrufen. Zur Untersuchung dieses Sachverhaltes wurden die täglichen Zunahmen im Intervall [10, 240], [240, 420] und [10, 420] sowie die Körpergewichte zum 240. und 420. Tag als Zuchtziele betrachtet (s. Tabelle 2).

Tabelle 2

Partielle Selektionserfolge (in kg) unter Verwendung von täglichen Zunahmen und von Körpergewichten als

\begin{tabular}{|c|c|c|c|c|c|c|c|}
\hline \multirow[b]{2}{*}{ Index } & \multirow[b]{2}{*}{$\mathbf{H}$} & \multicolumn{6}{|c|}{ partielle SE (mit $\left.d_{s}=1\right)$} \\
\hline & & $\Delta g_{10}$ & $\Delta \mathbf{g}_{240}$ & $\Delta \mathbf{g}_{420}$ & $\Delta \mathbf{g}_{\mathrm{TZ} 1}$ & $\Delta \mathbf{g}_{\mathrm{TZ2}}$ & $\Delta \mathbf{g}_{\mathrm{TZ}}$ \\
\hline $\mathrm{I}_{1}$ & $\overline{\mathbf{g}_{\mathrm{TZ1}}}$ & -0.52 & 12.42 & 18.49 & 0.0563 & 0.0377 & 0.0464 \\
\hline $\mathrm{I}_{2}$ & $\mathbf{g}_{\mathrm{TZ2}}$ & 2.24 & 9.38 & 20.38 & 0.0311 & 0.0611 & 0.0443 \\
\hline $\mathrm{I}_{3}$ & $\mathbf{g}_{\mathrm{TZ}}$ & 0.84 & 12.50 & 21.96 & 0.0507 & 0.0525 & 0.0515 \\
\hline $\mathrm{I}_{4}$ & $g_{240}$ & 5.31 & 15.61 & 22.22 & 0.0448 & 0.0367 & 0.0413 \\
\hline $\mathrm{I}_{5}$ & $g_{420}$ & 4.50 & 14.51 & 23.90 & 0.0436 & 0.0521 & 0.0473 \\
\hline
\end{tabular}
Zuchtziel (Genetic gains if daily gains or body weights are used as breeding goal)

mit $\mathrm{g}_{\mathrm{TZ1}}=\left(\mathrm{g}_{240}-\mathrm{g}_{10}\right) / 230 ; \mathrm{g}_{\mathrm{TZ2}}=\left(\mathrm{g}_{420}-\mathrm{g}_{240}\right) / 180$ und $\mathrm{g}_{\mathrm{TZ}}=\left(\mathrm{g}_{420}-\mathrm{g}_{10}\right) / 410$

Die Indizes $I_{1}, I_{2}$ und $I_{3}$ maximieren die tägliche Zunahme vom 10. bis zum 240. Tag, vom 240. bis zum 420. Tag und vom 10. bis zum 420. Tag. Die maximalen SE für die täglichen Zunahmen TZ1, TZ2 und TZ sind 0.0563, 0.0611 und 0.0515. Wird dagegen das Gewicht zum 420. Tag maximiert, so besitzen die partiellen SE für TZ1, TZ2 und TZ die Werte 0.0436, 0.0521 und 0.0473. Zur gleichzeitigen Berücksichtigung von Zunahmen und Körpergewichten im Zuchtziel bieten sich folgende Restriktionsvarianten an. 


$$
\begin{aligned}
& \Delta g_{420} \rightarrow \text { Max! bei a) } \Delta g_{240}=(1-\alpha) \cdot \Delta g_{240}^{*} \\
& \text { b) } \Delta g_{T Z 1}=\left(1-\alpha_{1}\right) \cdot \Delta g_{T Z 1}^{*} ; \quad \Delta g_{T Z 2}=\left(1-\alpha_{2}\right) \cdot \Delta g_{T Z 2}^{*} \\
& \text { c) } \Delta g_{T Z}=\left(1-\alpha_{3}\right) \cdot \Delta g_{T Z}^{*}
\end{aligned}
$$

In Darstellung (22) sind $\Delta g_{240}^{*}, \Delta g_{\mathrm{TZ1}}^{*}, \Delta g_{\mathrm{TZ2}}^{*}$ und $\Delta g_{\mathrm{TZ}}^{*}$ die maximal möglichen Werte für die zugehörigen SE ausgewiesen in Tabelle 2. Durch die Wahl von $\alpha, \alpha_{1}, \alpha_{2}$, und $\alpha_{3}$ wird eine vorgegebene prozentuale Reduzierung des maximalen Selektionserfolges erreicht. Die Vorgabe von $\alpha_{1}$ und $\alpha_{2}$ in Restriktionsvariante b) kann zu einem Optimierungsproblem führen, welches keine zulässige Lösung besitzt (vgl. Abschnitt 2.2, Formel (8), Bedingung (b)). Die partiellen SE für die Restriktionsvarianten a), b) und c) bei spezieller Wahl von $\alpha, \alpha_{1}, \alpha_{2}$, und $\alpha_{3}$ sind in Tabelle 3 zusammengestellt. Setzt man beispielsweise $\alpha_{1}=\alpha_{2}=0.10$, so besitzt Problem (22b) keine zulässige Lösung.

Tabelle 3

\begin{tabular}{|c|c|c|c|c|c|c|c|c|}
\hline \multirow[b]{2}{*}{ Index } & \multirow[b]{2}{*}{$\mathrm{H}$} & \multirow[b]{2}{*}{ Restriktion } & \multicolumn{6}{|c|}{ partielle SE $\left(\right.$ mit $\left._{\mathrm{s}}=1\right)$} \\
\hline & & & $\Delta g_{10}$ & $\Delta g_{240}$ & $\Delta g_{420}$ & $\Delta \mathbf{g}_{\mathrm{TZ1}}$ & $\Delta \mathrm{g}_{\mathrm{TZ} 2}$ & $\Delta \mathbf{g}_{\mathrm{TZ}}$ \\
\hline $\mathrm{I}_{4 \mathrm{R}}$ & $g_{420}$ & $\Delta g_{240}=0.98 \cdot \Delta g_{240}^{*}$ & 4.97 & 15.30 & 23.52 & 0.0449 & 0.0457 & 0.0543 \\
\hline$I_{5 R}$ & $\mathbf{g}_{420}$ & $\Delta g_{T Z}=0.95 \cdot \Delta g_{T Z}^{*}$ & 3.76 & 14.28 & 23.81 & 0.0457 & 0.0529 & 0.0489 \\
\hline $\mathrm{I}_{6 \mathrm{R}}$ & $\mathbf{g}_{420}$ & $\begin{aligned} \Delta g_{T Z 1} & =0.85 \cdot \Delta g_{T Z 1}^{*} \\
\Delta g_{T Z 2} & =0.85 \cdot \Delta g_{T Z 2}^{*}\end{aligned}$ & 3.28 & 14.29 & 23.64 & 0.0479 & 0.0519 & 0.0497 \\
\hline
\end{tabular}

Partielle Selektionserfolge (in kg) in Abhängigkeit vom Zuchtziel H und von den Restriktionen (Genetic gains depending on different breeding goals and restrictions)

mit $\Delta \mathrm{g}^{*}=$ maximaler SE gemäß Tabelle 2

Die Maximierung des Körpergewichtes zum 420. Tag unter der Bedingung, dass 98\% der maximal möglichen Veränderung im Körpergewicht zum 240. Tag erreicht wird reduziert den SE von 23.90 auf 23.52. Die Restriktionen für Indexvariante $\mathrm{I}_{6 \mathrm{R}}$ reduzieren die maximale Veränderung für das Gewicht zum 420. Tag von 23.90 auf 23.64. Zur Ableitung so genannter „restriktiver ökonomischer Gewichte“ wurden die Formeln (10) und (11) aus Abschnitt 2.2 benutzt (s. Tabelle 4).

Tabelle 4

Relative ökonomische und „restriktive ökonomische Gewichte“ im Zuchtziel für die Indexvarianten der Tabellen 2 und 3 („restrictive economic values“ of selection indexes from table 2 and 3)

\begin{tabular}{ccccccccc}
\hline & \multicolumn{3}{c}{ ohne Restriktionen } & \multicolumn{3}{c}{ mit Restriktionen* } \\
Gewichte & $\mathbf{I}_{\mathbf{1}}$ & $\mathbf{I}_{\mathbf{2}}$ & $\mathbf{I}_{\mathbf{3}}$ & $\mathbf{I}_{\mathbf{4}}$ & $\mathbf{I}_{\mathbf{5}}$ & $\mathbf{I}_{\mathbf{4 R}}$ & $\mathbf{I}_{\mathbf{5 R}}$ & $\mathbf{I}_{\mathbf{6 R}}$ \\
\hline $\mathbf{a}_{\mathbf{1 0}}$ & -1.0 & 0.0 & -1.0 & 0.0 & 0.0 & 0.0 & -0.2316 & -0.4212 \\
$\mathbf{a}_{\mathbf{2 4 0}}$ & 1.0 & -1.0 & 0.0 & 1.0 & 0.0 & 0.8075 & 0.0 & 0.1835 \\
$\mathbf{a}_{\mathbf{4 2 0}}$ & 0.0 & 1.0 & 1.0 & 0.0 & 1.0 & 0.5898 & 0.9728 & 0.8882 \\
\hline
\end{tabular}

$\left.\mathrm{H}=\mathrm{a}_{10} \cdot \mathrm{g}_{10}+\mathrm{a}_{240} \cdot \mathrm{g}_{240}+\mathrm{a}_{420} \cdot \mathrm{g}_{420} ; *\right)$ Normierung gemäß $\left.\|\mathrm{a}\|^{2}=\mathrm{a}^{\prime} \mathrm{a}=1\right)$

Verwendet man die in Tabelle 4 aufgeführten Koeffizienten als Wichtungsfaktoren im Zuchtziel, so ergeben sich die partiellen SE der restriktiven Indices aus Tabelle 3 ohne Berücksichtigung der entsprechenden Restriktionen.

\section{Diskussion}

Das Prinzip der Indexkonstruktion mit Restriktionen kann zur genetischen Veränderung der Gestalt der Wachstumskurve genutzt werden. Erfolgt die Schätzung tierspezifischer Zuchtwertkurven im RRM durch Polynome 4. Grades, so sind maximal 4 
untereinander und vom Zuchtziel unabhängige Restriktionen für die partiellen SE oder deren Linearkombinationen vorgebbar. Die in Abschnitt 2.2 bereitgestellten Formeln ermöglichen die Vorgabe von Restriktionen auf die partiellen SE zu bestimmten Zeitpunkten, um so zu gewünschten Veränderungen der Wachstumskurve zugelangen. Das Konzept der restriktiven Indizes kann ebenfalls genutzt werden, um Zuchtwerte für hohes Endgewicht bei zumindest suboptimaler Erhöhung der täglichen Zunahme abzuleiten. Im Ergebnis der Indexkonstruktion lassen sich „restriktive ökonomische Gewichte“ zu bestimmten Zeitpunkten des Wachstums angeben, so dass aus den Zuchtwertkurven pro Tier unmittelbar Einzelzuchtwerte für das Körpergewicht gebildet werden können.

Die Ableitung der SE erfolgte unter der Annahme, dass die zufälligen additiv-genetischen Regressionskoeffizienten und somit die Zuchtwertkurven pro Tier bekannt sind. Bei der herkömmlichen Indexkonstruktion wird dagegen von phänotypischen Informationsquellen, wie Vorfahren-, Eigen-, Geschwister- und Nachkommenleistungen ausgegangen und daraus für jeden Probanden ein Zuchtwert abgeleitet. Durch die Einbeziehung der numerischen Verwandtschaftsmatrix bei der Auswertung mit einem RRM beruhen die geschätzten Zuchtwertkurven bereits auf allen phänotypischen Informationsquellen, die für den jeweiligen Kandidaten der Selektion zur Verfügung stehen. Deshalb ist es wenig sinnvoll mit Hilfe der Matrix K unter zusätzlicher Verwendung der umweltbedingten Kovarianzmatrix eine Indexkonstruktion beispielsweise basierend auf Eigenleistungen durchzuführen. In dieser Arbeit werden die genetischen Effekte pro Tier zu jedem Zeitpunkt als gegeben vorausgesetzt. Folglich besitzen die Vorhersagen der SE nur marginale Bedeutung. Zur Beurteilung verschiedener Restriktionsvarianten sind nicht die absoluten Werte sondern die Verhältnisse der SE zu verschiedenen Zeitpunkten untereinander sowie die Anteile der partiellen SE an den maximal möglichen SE von vorrangiger Bedeutung. Durch die Ableitung zugehöriger „restriktiver ökonomischer Gewichte“ gelingt es aus den Zuchtwertkurven für jedes Tier Einzelzuchtwerte zu gewinnen auf deren Basis eine Rangordnung durchgeführt werden kann. Eine derartige Vorgehensweise schließt natürlich nicht aus, dass nach einer Vorauswahl nochmals die Zuchtwertkurven einzelner Probanden für den endgültigen Selektionsentscheid herangezogen werden.

\section{Literatur}

ALBUQUERQUE, L.G.; MEYER, K.:

Estimates of covariance functions for growth from birth to 630 days of age in Nelore cattle. J. Anim. Sci. 79 (2001), 2776-2789

ARANGO, J.A.; CUNDIFF, L.V.; VAN VLECK, L.D.:

Genetic parameters for weight, weight adjusted for body condition score, high, and body condition score in beef cows. J. Anim. Sci. 80 (2002), 3112-3122

CUNNINGHAM, E.P.; MOEN, R.A.; GJEDREM, T.:

Restriction of selection indexes. Biometrics 26 (1970), 67-74

HARVILLE, D.A.:

Optimal procedures for some constrained selection problems. J. of American Statistical Association 69 (1974), 446-452

HAZEL, L.N.:

The genetic basis for constructing selection indexes. Genetics 28 (1943), 476-490

JAMROZIK, J.; SCHAEFFER, L.R.:

Estimates of Genetic Parameters for a Test Day Model with Random Regressions for Yield Traits of First Lactation Holsteins. J. Dairy Sci. 80 (1997), 762-770

KEMPTHORNE, O.; NORDSKOG, A.W.:

Restricted selection indices. Biometrics 15 (1959), 10-19 
KIRKPATRICK, M.; LOFSVOLD, D.; BULMER, M.:

Analysis of inheritance, selection and evolution of growth trajectories. Genetics 124 (1990), 979-993

KREJČOVÁ, H.; MIELENZ, N.; PŘIBYL, J.; SCHÜLER, L.:

Estimation of genetic parameters for daily gains of bulls with multi-trait and random regression models. Arch. Tierz., Dummerstorf. 50 (2007a), 37-46

KREJČOVÁ, H.; MIELENZ, N.; PŘIBYL, J.; SCHÜLER, L.:

Comparison of breeding values for daily gains of bulls estimated with multi-trait and random regression models. Arch. Tierz., Dummerstorf. 50 (2007b), 147-154

LIN, C.Y.; TOGASHI, K.:

Maximization of lactation milk production without decreasing persistency. J. Dairy Sci. 88 (2005), 2975-2980

MATTHES, H.-D.; RUDOLPH, P.E.; HERRENDÖRFER, G.:

Verwendung von Wachstumsfunktionen zur Charakterisierung des Wachstums von Fleischrindrassen. Arch. Tierz., Dummerstorf. 39 (1996), 121-127

MEYER, K.:

Estimates of genetic and phenotypic covariance functions for postweaning growth and mature weight of beef cows. J. Anim. Genet. 116 (1999), 181-205

MEYER, K.:

Estimates of direct and maternal covariance functions for growth of Australian beef calves from birth to weaning. Genet. Sel. Evol. 33 (2001), 487-514

NIEBEL, E.; VAN FLECK, L.D.:

Optimal procedure for restricted selection indexes. Z. Tierzüchtg. Züchtungsbiologie 100 (1983), 9-26

SCHAEFFER, L.R.; DEKKERS, J.C.M.:

Random regressions in animal models for test-day production in dairy cattle.” Proc. $5^{\text {th }}$ WCGALP, Guelph, Canada (1994)

SWALVE, H.H.; GUO, Z.:

An illustration of lactation curves stratified by lactation yields within herds. Arch. Tierz., Dummerstorf. 42 (1999), 515-525

TOGASHI, K.; LIN, C.Y.:

Selection for milk production and persistency using eigenvectors of random regression coefficient matrix. J. Dairy Sci. 89 (2006), 4866-4873

Eingegangen: 22.03.2007

Akzeptiert: 30.08.2007

Autor für Korrespondenz

Dr. NORBERT MIELENZ

Institut für Agrar- und Ernährungswissenschaften

der Martin-Luther-Universität Halle-Wittenberg

Adam-Kuckhoff-Straße 35, D-06108 Halle

E-Mail: norbert.mielenz@landw.uni-halle.de 